\title{
Models of Intergenerational and Transgenerational Transmission of Risk for Psychopathology in Mice
}

\author{
Torsten Klengel ${ }^{1,2,4}$, Brian G Dias ${ }^{2,4}$ and Kerry J Ressler ${ }^{\star, 1,2,3}$ \\ ${ }^{1}$ Department of Psychiatry, Harvard Medical School, McLean Hospital, Belmont, MA, USA; ${ }^{2}$ Department of Psychiatry and \\ Behavioral Sciences and Yerkes National Primate Research Center, Emory University, Atlanta, GA, USA; ${ }^{3}$ Howard Hughes \\ Medical Institute, Chevy Chase, MD, USA
}

Trajectories toward risk or resilience in psychiatric disorders are influenced by acquired and inherited factors. More recently, evidence from rodent studies suggest that acquired risk factors can be transmitted through non-genomic, epigenetic mechanisms to subsequent generations, potentially contributing to a cycle of disease and disease risk. Here, we review examples of transmission of environmental factors across generations and illustrate the difference between behavioral transmission and epigenetic inheritance. We highlight essential definitions of intergenerational and transgenerational transmission of disease risk with corresponding examples. We then explore how these phenomena may influence our understanding of psychiatric disorders leading toward new prevention and therapeutic approaches.

Neuropsychopharmacology Reviews (2016) 41, 2I9-231; doi: I0.1038/npp.20I5.249; published online 9 September 2015

\section{INTRODUCTION}

Stress-related psychiatric disorders such as major depression, posttraumatic stress disorder (PTSD), and other anxiety disorders are highly prevalent in the general population (Kessler et al, 2005), with an even higher prevalence in military and socioeconomically disadvantaged populations (Hoge et al, 2004; Gillespie et al, 2009). The pervasiveness of these disorders is increasing in the public perception and recent studies support this view, although there is some controversy with regard to the underlying causes (Heimberg et al, 2000; Kessler et al, 2005; Compton et al, 2006; Hidaka, 2012). Changing environmental conditions with respect to not only socioeconomic status, social interactions, trauma exposure, and workload, but also nutritional status, may contribute to this phenomenon and thus potentially lead to a self-reinforcing risk for disease. Additionally, stress-related disorders demand increasing medical and economical resources and are predicted to become the leading cause of disability worldwide in the near future (Wittchen et al, 2011; Whiteford et al, 2013).

Our understanding of the molecular mechanisms of risk and resilience for disease is pivotal for the development of effective preventative and therapeutic strategies that are

${ }^{*}$ Correspondence: Dr KJ Ressler, Department of Psychiatry, Harvard Medical School, McLean Hospital, 115 Mill Street, Belmont, MA 02478, USA, Tel: +1 404727 7739, Fax: +1 404727 8070,

E-mail: kressler@mclean.harvard.edu

${ }^{4}$ These authors contributed equally to this manuscript.

Received 6 April 2015; revised 3 August 2015; accepted 12 August 2015; accepted article preview online 18 August 2015 superior to the currently available limited therapies. It is widely accepted that environmental and genetic factors contribute to the development of psychiatric disorders. Although much effort is spent on studies investigating the genetic basis of psychiatric disorders and outstanding emerging studies point toward a polygenic risk to disease (Schizophrenia Working Group of the Psychiatric Genomics Consortium, 2014), the overall contribution of genetic factors appears to be rather small in particular for stress and fear disorders based on our current knowledge (Major Depressive Disorder Working Group of the Psychiatric GWAS Consortium, 2012; Sullivan et al, 2012; Wray et al, 2012; Lee et al, 2013; Levinson et al, 2014). In contrast to the successful genetic studies for schizophrenia or bipolar disorder (Schizophrenia Working Group of the Psychiatric Genomics Consortium, 2014; Muhleisen et al, 2014), studies on depression so far have failed to identify reliable genetic markers at sample sizes at which first loci in schizophrenia were identified (Major Depressive Disorder Working Group of the Psychiatric GWAS Consortium, 2012). The genomics of PTSD is more nascent, and it is too early to tell which trajectory this disorder will have with regards to genetic contribution, though twin studies suggest up to $40 \%$ heritability. Additionally, several other moderately powered GWAS have begun to identify genome-wide level significant findings (Guffanti et al, 2013; Logue et al, 2013; Nievergelt et al, 2015). The underlying reasons likely involve a larger genetic, but also phenotypic, heterogeneity and a stronger influence of the environment (Levinson et al, 2014). Alternative models such as gene by environment interaction studies take this dual influence into account and 
may provide more insight into the pathophysiology of these disorders in the future (Klengel and Binder, 2013a; Manuck and McCaffery, 2014; Zannas, Wiechmann, Gassen and Binder, this issue).

Environmental factors influencing the long-term risk for psychiatric disorders have been known for centuries. Such factors have regained attention in the post-genomic era in recent years. These factors can exert a profound influence on disease trajectories when they occur during vulnerable periods in brain development like in utero development and early life until completion of adolescence, subsequently increasing the risk for mental health problems in adulthood (Kessler et al, 1997; Kendler et al, 2000). Human studies using a cross-sectional design can and have investigated the overall influence of severe stress or childhood abuse on the risk for psychiatric disorders without having access to neuronal tissue for further analyses. However, animal models, in contrast, provide valuable insights into the molecular mechanisms of how the environment contributes to risk and resilience. Besides direct exposure to stress both in utero and in early life, recent studies in rodents also point toward a transmission, and even inheritance, of environmentally accumulated risk factors via the germline that may occur prior to conception. Epigenetic mechanisms of gene regulation have been posited as being critical to such transmission and inheritance.

In the remainder of this review, we select examples from studies in rodents modeling the intergenerational and transgenerational transmission of environmentally induced phenotypes with regard to psychiatric phenotypes. We describe potential molecular mechanisms in the form of epigenetic modifications that may underlie the transmission and inheritance of traits and highlight the current controversy regarding these concepts.

\section{THE DEFINITION OF INTERGENERATIONAL AND TRANSGENERATIONAL TRANSMISSION AND INHERITANCE}

Pivotal to the understanding of the transmission of risk for psychopathology across generations, in both humans and rodent models, is the definition of intergenerational vs transgenerational transmission and the implications for their underlying molecular mechanisms. In addition, it is important to differentiate between a behavioral or social transmission and inheritance through the germ cells.

Intergenerational transmission of phenotypes relevant to psychiatric disorders is common in humans and mouse models. It implies the direct exposure to the stressor of the parental (F0) and subsequent generation (F1) by means of the developing germ cells or fetus. When exposure occurs before conception, male and female germ cells are equally exposed and thus, may result in an intergenerational transfer (Figure 1). An exception to this is the exposure of the developing fetus in utero or postnatal offspring to any environmental condition. Here, exposure occurs not only to the fetus or young offspring (F1) but also to the developing germ cells in this generation, resulting in an intergenerational transmission to the F2 generation (Figure 1). A transgenerational transmission is present when effects of the ancestral exposure to an environment during pregnancy are still present in the F3 generation, the first generation that is not directly exposed. In contrast, exposure before conception would indicate a true transgenerational inheritance in the F2 generation (Skinner, 2008; Dias and Ressler, 2014a).

A behavioral or social transmission implies the conscious or unconscious transfer of information through the behavior of the ancestral generation to the offspring. This will occur inevitably when the parental generation interacts with the offspring and only cross-fostering, in vitro fertilization, or experiments across multiple generations can control for this effect. In humans, this is virtually impossible to achieve for ethical and practical reasons, although adoption studies can provide some insight. In contrast to a behavioral or social transmission of information, the inheritance through the germ cells is independent of the direct behavior of the ancestral generation to the offspring. Here, information is transmitted through molecular means, and recent studies suggest that epigenetic mechanisms are implicated in the inheritance of environmentally acquired information (Figure 2). Epigenetic mechanisms in general influence transcriptional regulation, and we briefly describe the main features below and in Text Box 1. Pivotal to normal somatic but also germ cell development, epigenetic marks regulate transcriptional processes in a time-dependent and cell type-dependent manner. Importantly, environmental signals can influence regular epigenetic signaling thereby influencing epigenetic pattern, a process that is termed epigenetic programming. Although we focus on the detrimental effects of early life stress and abuse in this review, epigenetic programming per se is a normative process that occurs in development and adulthood, and is but one mechanistic avenue that is impacted equally by positive and negative environmental stimuli. Although there are many animal studies that are attempting to understand the role of epigenetics in the inheritance and transmission of traits across generations, it is debated whether and to what extent such mechanisms operate in mammalian species including humans and how these compare and contrast with more accepted transgenerational inheritance mechanisms that have been worked out in plants (Daxinger and Whitelaw, 2012; Heard and Martienssen, 2014). The most prominent examples with regard to psychiatric phenotypes to date investigate either severe stressors and/or signals relevant in an evolutionary context (Franklin et al, 2010; Morgan and Bale, 2011; Dietz et al, 2011; Rodgers et al, 2013; Gapp et al, 2014; Dias and Ressler, 2014b). It is highly unlikely that all environmental events impact epigenetic signatures of genes in the gametes to elicit intergenerational and transgenerational phenotypes. Therefore, any discussion 


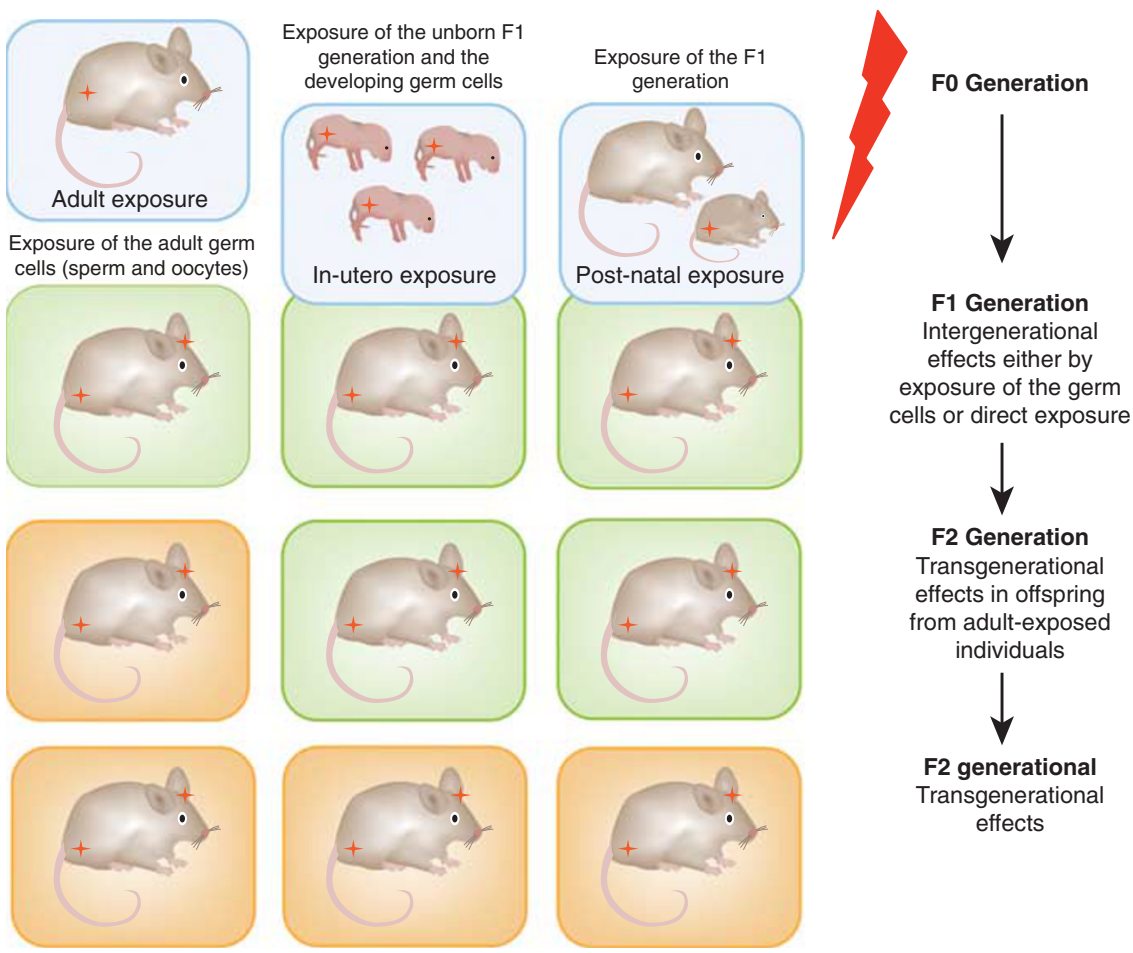

Figure 1. Environmental conditions can influence decent generations by distinct modes. The first column represents the exposure of an adult rodent to a stressful or aversive environment not only affecting the neural system of the exposed generation but also the germ cell through direct exposure. The germ cells give rise to the F1 generation representing an intergenerational transmission. The germ cells of the F1 generation are not exposed to the stressor and generate the subsequent F2 generation that represents a transgenerational transmission, which is not exposed to the stressor by means of a direct exposure or exposure of the germ cells. The second column represents the in utero exposure of a developing F1 generation to an environmental factor. This generation and the F2 generation, by means of the developing germ cells, are directly exposed, thus, representing an intergenerational transmission. The F3 generation represents the first generation that was not exposed to the stressor representing a transgenerational transmission. The third column represents the exposure of a F1 generation during postnatal development. Similar to the second example, this F1 as well as the F2 generation is directly exposed and therefore represent intergenerational transmission, with the following F3 generation representing a transgenerational transmission.

of the inheritance of traits across generations via epigenetic mechanisms should be tempered by our limited understanding of the extent to which environmental signals leave epigenetic marks on the genome that are then transmitted to subsequent generations via the gametes.

\section{EXAMPLES OF HUMAN STUDIES SUGGESTING INTERGENERATIONAL AND TRANSGENERATIONAL TRANSMISSION OF SOME ACQUIRED PHENOTYPES}

Some interesting studies on well-characterized human cohorts across generations have found evidence for the influence of the ancestral environment on subsequent generations. An important example is the Dutch hunger winter in World War II. Here, exposure of pregnant women to a severe famine led to a higher risk for metabolic and mental health diseases in the (in utero exposed) F1 generation. Intriguingly, it was found that the risk was increased when exposed in the first half of the pregnancy, but the risk appeared to be lower when the fetus was exposed during the last trimester. The F1 generation, raised under normal conditions after the war, gave rise to a subsequent F2 generation (that was also exposed by means of the developing germ cells) that again showed an increased risk for metabolic disorders but only in children from at-risk F1 fathers (Kaati et al, 2007). Later studies underscored the contribution of this severe environmental condition during the war toward DNA methylation differences in whole blood present in subsequent generations (Heijmans et al, 2008; Tobi et al, 2014). Of note, DNA methylation was altered when women were exposed very early in pregnancy but not in late gestation. However, it remains an open question if this holds true for the F3 generation that would be the first unexposed generation, pointing toward an epigenetic inheritance.

Additional studies, see for example Yehuda et al, reviewed below, have found poignant examples of intergenerational transmission of risk following trauma exposure, particularly with regards to offspring of the Holocaust. The remainder of this review highlights behavioral paradigms and complementary epigenetic mechanisms that are necessary in the understanding of intergenerational and transgenerational transmission and inheritance. 


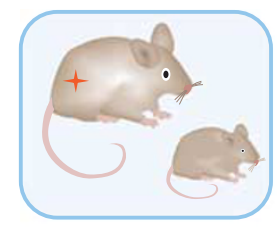

Biological transmission

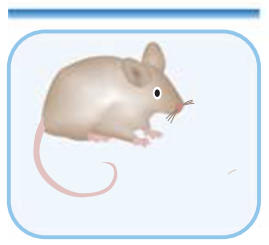

Proof by

- In-vitro fertilization

- Cross fostering paradigms

- Multiple generation paradigm breeding to F3

resulting in a prevention of direct

contact and thus behavioral influence of the $\mathrm{F} 0$ with the $\mathrm{F} 1$ generation

Figure 2. Transmission by behavioral or social cues is distinct from biological inheritance. The biological inheritance of acquired information through epigenetic mechanisms can be distinguished from the behavioral transmission in controlled experiments. Here, the contact of the stressexposed father to the offspring can be excluded though in vitro fertilization (IVF) that also prevents effects on the female animal during mating potentially influencing maternal care. Other possibilities are cross-fostering paradigms and breeding schemes to the F3 generation that limit the potential of a behavioral transmission of information. This mode of information transfer is common and inevitable when parental generations interact with the offspring. Here, behavioral alterations can induce epigenetic modifications that are not present when animals are conceived through IVF, for example.

\section{INFLUENCE OF POSTNATAL ENVIRONMENT ON BIOLOGY OF FUTURE GENERATIONS}

The postnatal environment is crucial for the development of mammals, with a strong impact of perturbations during this period on later life health outcomes. Rodent models of postnatal environmental stressors gained great attention because of the implication of epigenetic mechanisms underlying the long-lasting biological programming through early life stress. One of the most prominent examples investigates the effect of naturally occurring differences in maternal care in rats on the offspring's stress response in adulthood (Weaver et al, 2004). Differences in the first week of life, with regard to maternal care, program the molecular and behavioral response of the hypothalamus-pituitary-adrenal (HPA) axis to stress in adulthood (Zhang et al, 2013). On the molecular level, variation in maternal care of the rat dam to the offspring results in divergent expression and translation of the glucocorticoid receptor (GR). In this case, offspring receiving a high amount of maternal care exhibit a stronger
BOX 1 Epigenetic Modifications Responsive to Environmental Signals

Histone modification: The DNA strand needs to be highly condensed into chromosomes, which is facilitated by wrapping the DNA around core histone proteins forming the nucleosome and thereby allowing the DNA to fit into the nucleus. To regulate the accessibility of important functional regions of the DNA strand to transcriptional regulators, histone proteins can comprise a plethora of modifications predominantly at the $\mathrm{N}$-terminal tails that alter the spatial structure of the chromatin and the interaction of DNA-binding proteins leading to an increase or decrease of transcription (lenuwein and Allis, 200 I).

DNA methylation and related chemical modifications: DNA methylation is the addition of a methyl group to most often the nucleotide cytosine, but also to other nucleotide residues. These modifications influence how proteins bind to the DNA leading to an increased or decreased transcription depending on their genomic location. More recent work shows that there are modifications beyond the classical 5-methyl cytosine modification with 5-hydroxymethyl cytosine as potentially important modification in neuronal tissue (Szulwach et al, 20I I). Non-coding RNA: Besides protein-coding RNAs, non-coding RNAs have a major role in the regulation of gene transcription (Mercer and Mattick, 20 I3; Morris and Mattick, 2014). Non-coding RNAs include: micro RNAs (miRNAs), piwiinteracting RNAs (piRNAs), and long non-coding RNAs (IncRNAs), that can interfere with transcriptional regulation and translation on multiple levels. For a long time, epigenetic modifications, especially DNA methylation, have been considered irreversible, defining cellular identity in a multicellular organism. More recently, it has been shown that even stable chemical modifications such as DNA methylation underlie highly dynamic regulation with the potential of reversibility, making these mechanisms suitable for encoding the long-term impact of the environment also in post-mitotic tissue such as neurons (Kriaucionis and Heintz, 2009; Ma et al, 2009; Sweatt, 2013). Although depicted separately for clarity, epigenetic mechanisms form a complex interactive network with joint activities of different mechanisms contributing to the molecular outcome (Murr, 2010). DNA methylation and histone modification are strongly interconnected and may serve as platforms for non-coding RNA binding.

GR presence in corticolimbic structures, leading to an enhanced negative feedback of the HPA axis in response to stress. The stimulation of the offspring by tactile input appears to be the critical environmental signal that has been suggested to lead to an increased serotonergic signaling in the hippocampus, and in turn, to an increased expression of the transcription factor NGFI-A via cAMP-dependent mechanisms. The increased expression of this transcription factor leads to an increased binding to the NGFI-A containing neuron-specific exon $1_{7}$ sequence of the GR promoter in the hippocampus and subsequent activation of transcription. Together, these processes result in the increased stress-dependent HPA negative feedback seen as a result of higher maternal care.

Subsequent studies investigating the long-lasting effects of high $v s$ low maternal care focused on DNA methylation as a mechanism underlying programming of the long-term effects of maternal care. The results indicated that high maternal care is associated with lower methylation in particular at one $\mathrm{CpG}$ at the NGFI-A binding site compared with low maternal care that led to an increased methylation, which at least in part, is mediated by increasing levels of hydroxymethylation (Zhang et al, 2013). The elevated DNA methylation levels in the low maternal care offspring negatively influence the binding of the NGFI-A to its binding site and thus provide a mechanism for a reduced transcriptional activation of the GR. These results on the level of DNA methylation are paralleled by changes in histone modification patterns at this locus. Here, increased 


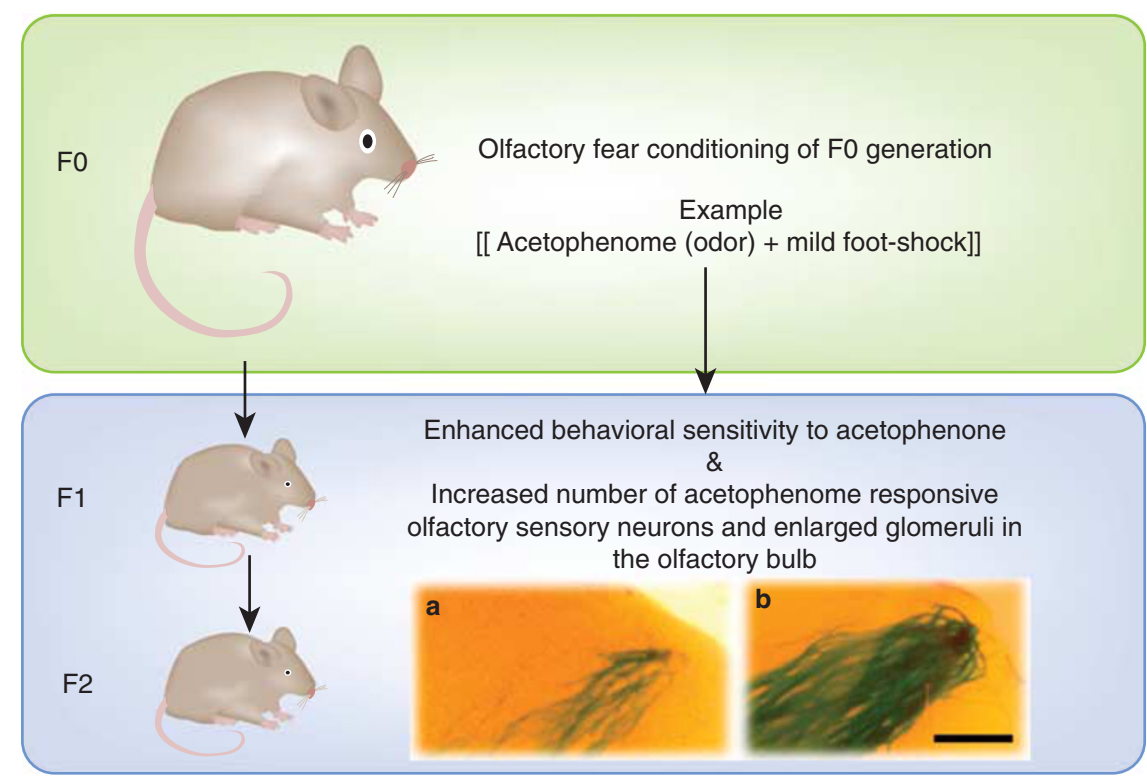

Figure 3. Transgenerational inheritance of enhanced olfactory sensitivity and its neuroanatomical representation. In this example, transgenerational inheritance is shown for Odor A (eg, acetophenone) in F1 and F2 generation of mice after the F0 generation was fear-conditioned to Odor A. Exposing an F0 generation of mice to an olfactory fear-conditioning paradigm, wherein presentations of a particular odor (eg, Odor A) are paired with mild foot-shocks, results in a sensitivity toward that odor in the F1 and F2 generations that have never previously been exposed to Odor A. When acetophenone, an odorant that activates M71-expressing odorant receptors in the nose, is used as the conditioning odor in the F0 generation, there are more M71-expressing olfactory sensory neurons in the nose of these animals. This enhanced neuroanatomical representation for M71 is also observed in the noses and olfactory bulbs of the F1 and F2 generation. DNA methylation is altered around the M71 receptor gene in sperm of the F0 and F1 generations, potentially associated with the greater number of M71 neurons in the F1 and F2 generations.

H3K9 acetylation and H3K4 methylation in offspring receiving high maternal care compared with low maternal care lead to increased transcriptional activity (Weaver et al, 2004). In particular, histone modifications are suggested to repress the binding of enzymes that methylate DNA in a de novo manner. The effect of maternal care is not restricted to the GR locus, although the molecular mechanisms leading to behavioral changes are best elaborated at this locus. However, the effects are likely genome wide with both increased and decreased DNA methylation patterns across larger stretches of DNA, which is similar to what is seen in human post-mortem tissue (McGowan et al, 2011; Suderman et al, 2012).

In summary, this example shows the long-lasting effect of early life environments with the influence of the ancestral generation on stress reactivity in the offspring through an epigenetic mechanism. Moreover, the dam's behavior to the offspring is priming their own behavior toward the granddaughter generation, with rats raised by high maternal care mothers in turn being more likely to exhibit high levels of maternal care. Cross-fostering experiments with low maternal care animals revealed that this phenomenon is most likely transmitted through behavior and less likely by means of epigenetic inheritance (Francis et al, 1999; Champagne, 2008). Together, these findings suggest that the maternal behavior in early life induces epigenetic modifications that in turn regulate responsiveness to stress, complementing learned maternal behavior toward the following generation thus reinforcing behavioral traits via epigenetic mechanisms across generations without the element of inheritance.

The MSUS model, which includes unpredictable maternal separation combined with unpredictable maternal stress, exemplifies effects of stress in early life that are inherited to subsequent generations through the gametes (Franklin et al, 2010; Gapp et al, 2014). In this model, unpredictable maternal separation for $3 \mathrm{~h}$ daily from postnatal day 1 to 14 was combined with unpredictable maternal stress resulting in depressive-like behaviors and an altered behavioral response to aversive environments in male adult animals comprising the F1 generation. This generation is then bred to naïve females and removed shortly after conception to avoid direct effects of the F1 generation on the subsequent F2 generation. Similar, F2 animals are bred again to result in an F3 generation that represents the first generation that is not exposed to the MSUS paradigm. Depressive-like behavior, as scored by the forced swim test, was seen in the F2 female and F3 male generation suggesting sex-specific effects that skip generations. Simultaneously, DNA methylation changes in candidate promoter regions in sperm of F1 males were observed that correlated with corresponding DNA methylation and gene expression changes in the brains of F2 animals (Franklin et al, 2010). In a recent follow-up, the authors demonstrated that MSUS F1 and F2 males were less anxious as assessed by the elevated plus maze and the light-dark Text Box 1. In addition, both generations showed alterations of insulin and glucose 
metabolism. Of note, both the F1 generation through direct exposure, and the F2 generation through exposure of germ cells, were exposed to the MSUS paradigm. However, the authors went on and investigated the possibility that small non-coding RNA may contribute to the transmission of the phenotype to the F2 generation by deep sequencing of F1 sperm RNA. They found several microRNAs (miRNAs) upregulated and a piRNA cluster downregulated, and they confirmed the differential expression of five candidate miRNAs. Although the authors reported miRNA regulation in F2 brain and serum, the results were negative for F2 sperm and miRNA regulation in the $\mathrm{F} 3$ generation, suggesting an alternative mode of transmission from the F2 to the F3 generation. Making a strong case for the importance of sperm miRNAs in modulating the physiology and behavior of the next generation, the authors purified and injected RNA from F1 sperm into fertilized wild-type oocytes, leading to behavioral and metabolic effects that were comparable in some respects with F2 offspring generated from F1 MSUS males via traditional mating.

\section{INFLUENCE OF ENVIRONMENT IN UTERO ON BIOLOGY OF FUTURE GENERATIONS}

Gestating embryos in utero are particularly vulnerable to environmental perturbations that the maternal lineage may be exposed to. Such perturbations can have profound effects on the development of neuropsychiatric disorders like depression, anxiety, and PTSD. For example, antenatal depression occurring during pregnancy increases the future risk of depression in the gestating offspring and is hypothesized to be a result of alterations in HPA axis function (Goodman and Gotlib, 1999). Similarly, exposure of the maternal lineage to adverse life events has been correlated with the development of schizophrenia and autism in the next generation (van Os and Selten, 1998; Khashan et al, 2008; Kinney et al, 2008).

One of the most striking examples of the influence of the in utero environment on the biology of the gestating generation in humans comes from the work of Yehuda and colleagues (Yehuda et al, 2005). Their work has demonstrated that exposure of pregnant women to the 11 September 2001 terrorist attacks profoundly affected HPA axis function in the gestating babies at a timepoint as early as 1 year of age. Babies that were in utero at the time that their mothers directly experienced the $9 / 11$ attacks (and who consequently developed PTSD as a result of this exposure) had lower cortisol levels at age 1, compared with a comparable cohort of babies whose mothers had not experienced these attacks. Such influences of maternal PTSD on HPA axis function have been reported in another intergenerational population; the offspring of the Holocaust survivors (Yehuda et al, 2008). For example, maternal PTSD as a result of the Holocaust was associated with poorer emotional and physical health and higher use of psychotropic medications in adult offspring (Flory et al, 2011). Most related to the effect of ancestral Holocaust exposure on HPA function in the descendant offspring are the results showing differential methylation of the GR and the $11 \beta$-hydroxysteroid dehydrogenase type 2 activity in these offspring as adults (Yehuda et al, 2014; Bierer et al, 2014). It remains an open question, how the exposure and the subsequent development of PTSD in the parental generation influences HPA axis function and disease development in the offspring largely owing to the fact that it is impossible to disentangle the biological effects of the exposure to traumatic experiences in the womb from the behavioral component of the diseased mother interacting with the child.

Preclinical work using rodent systems to model such influences have subjected the pregnant mouse or rat to specific environmental cues as well as broad stressors and then tested the F1 generations' physiology and behavior toward these cues. For example, in utero exposure to a maternal diet supplemented with 'cherry' or 'mint' odors results in the descendant F1 generation showing a preference for those odors. Accompanying this behavioral preference was increased volumes in the olfactory bulbs of the glomeruli that process cherry (M71-expressing olfactory sensory neurons and glomeruli) and mint (M72-expressing cells and glomeruli) (Todrank et al, 2011). In other studies, prenatal exposure to cocaine affected the F1 generation's behavior toward cocaine, as well as dopaminergic neurotransmission and stress responsiveness (Malanga et al, 2007; Wang et al, 2013). Additionally, exposure of pregnant rat dams to a fungicide, Vinclozolin, has been shown to affect reproductive and behavioral endpoints in several generations removed from this F0 exposure (Crews et al, 2007; Skinner et al, 2008; Gillette et al, 2014).

From a mechanistic viewpoint, in utero perturbations are thought to converge on HPA axis and glucocorticoid signaling pathways to exert their effect on the F1 and subsequent generations (reviewed in Moisiadis and Matthews, 2014a, b). Focusing on rodents, antenatal excess exposure to glucocorticoids has resulted in offspring that showed enhanced corticosterone dynamics in response to stress in adulthood (Nagano et al, 2008). Although the developing fetus is protected by the placenta barrier against glucocorticoid-induced apoptotic and growth-inhibiting effects, the excess glucocorticoid exposure through severe stress might saturate this system (Seckl and Meaney, 2006). In addition, deficits in learning and memory, and increased anxiety, have been observed in such instances (Hauser et al, 2009). Accompanying such behavioral alterations are structural changes in brain regions including the hippocampus, amygdala, and bed nucleus of the stria terminalis, that are known to be involved in learning, memory, anxiety, and stress responsiveness (Noorlander et al, 2008; Oliveira et al, 2012; Zuloaga et al, 2012). Complementing these studies with glucocorticoid exposure are others that subjected pregnant rats to environmental stressors at different points in gestation. These in utero stressors have been shown to affect oxytocin receptor levels, quality of maternal care, stress responsiveness, and anxiety in multiple litters (Champagne 
and Meaney, 2006), as well as learning and memory, HPA axis function, sensitivity to SSRIs, CRF, and GR expression in the brain (Mueller and Bale, 2007, 2008). Sex-specific epigenetic regulation of gene expression in the placenta has also been observed after such manipulations and presents an avenue via which offspring biology extending into the F2 generation could be affected by in utero experiences (Mueller and Bale, 2008, Morgan and Bale, 2011).

Studies like these (and those not included owing to space constraints) paint a picture that highlights the importance of experiences within the womb as powerful determinants of physiology and behavior for many generations to come. Comparing and contrasting the effects of exogenously administered stress hormones $v s$ environmental stressors will undoubteduly illuminate crucial convergent pathways by which in utero experiences might affect future populations.

\section{INFLUENCE OF ENVIRONMENT PRIOR TO CONCEPTION ON BIOLOGY OF FUTURE GENERATIONS}

Environmental perturbations to which paternal lineages are exposed can have significant influences on future generations, as demonstrated both in human observational studies and rodent manipulation experiments. The Dutch hunger winter studies, mentioned above, provide an example of this. Animal models, using an F0 generation with environmental exposure prior to conception, have begun to examine the contributions to descendant biology of environmental perturbations in prior generations. In keeping with the impact of pre-conceptional nutrition of an F0 animal population on F1 metabolism, studies have shown that a high-fat diet administered to male F0 rats prior to and during mating results in profound effects on F1 female offspring ( $\mathrm{Ng}$ et al, 2010). These include alterations in pancreatic physiology and gene expression, insulin sensitivity, and glucose tolerance. In another study that manipulated the paternal diet in mice by administering a low protein diet, gene expression in the liver and cholesterol metabolism was affected in F1 offspring (Carone et al, 2010). More recently, attention is being paid to the effect of subjecting F0 animals to behavioral and environmental stressors and then examining the influences of such perturbations on the behavior and biology of descendant generations.

Dietz and colleagues (Dietz et al, 2011) subjected F0 male mice to chronic social defeat stress. They then allowed for mating with control females, subsequently assaying behavior and physiology in the F1 generation. Both male and female F1 mice sired by F0 males that had been socially defeated showed higher baseline corticosterone levels, as well as decreased levels of vascular endothelial growth factor, compared with the F1 animals sired by control males. Significant elevations in anxiety- and depression-like metrics were also noted in these animals compared with controls. To address whether these effects were inherited, the authors used sperm from socially defeated and control males to perform IVF. This experiment yielded some data to suggest that the aforementioned behavioral effects might well be inherited, but the overwhelming data suggested this not to be the case. This led the authors to conclude that the observed effects were not truly inherited but instead possibly transmitted from the F0 female as a consequence of information she may have received about having mated with an 'impaired' male.

In a similar study, Bale and colleagues (Rodgers et al, 2013) exposed male mice to 6 weeks of chronic variable stress during puberty or in adulthood, and then tested subsequently conceived F1 males and females for dynamics of stress responsiveness, behavior, and gene expression in specific brain nuclei. Exposure to such chronic variable stress resulted in offspring that had lowered responsiveness of the HPA axis complemented by changes in the expression of genes relevant to such responsiveness in the paraventricular nucleus of the hypothalamus and the bed nucleus of the stria terminalis. The authors then extended their findings from a phenomenological perspective to one that took a first step toward ascribing mechanism to their findings. Querying the miRNA content in the F0 sperm yielded data indicating that specific miRNA are regulated in the sperm of these F0 stressed males and that robust gene expression changes were detected in the offspring paraventricular nucleus and bed nucleus of the stria terminalis. Ongoing, unpublished data from these authors are revealing an unprecedented level of functionality of these miRNA. More specifically, injecting these individual miRNA into single cell zygotes recapitulates the previously observed effects (Rodgers et al, 2014) similar to the study by Gapp et al that suggests that small RNAs in sperm are potential signals of inheritance (Gapp et al, 2014). Studies like these are at the forefront of establishing a crucial role for non-coding RNA, such as miRNA, as the conduits of information inheritance across generations.

Further evidence for the effect of F0 exposure to environmental features altering how future generations respond to those features are provided from recent studies in the olfactory modality (Dias and Ressler, 2014b). We subjected adult male F0 mice to an olfactory fearconditioning paradigm, pairing mild foot-shocks with discrete, neutral odors like acetophenone or propanol, which have known odorant receptor genes. This results in the F0 generation exhibiting fear-like behavior toward the odor that it was trained to. By allowing mating 10 days after the last day of conditioning, and separating the mating pair 12 days later, we were able to ask how the subsequently conceived F1 generation perceives the odor that their F0 father was conditioned with, despite the F1 animals having no prior exposure to this odor. At a behavioral level, we found an enhanced sensitivity to this odor using an Odor Potentiated Startle assay as well as by measuring association time with various concentrations of the odors. This increase in sensitivity was specific to the odor used to condition the F0 generation, suggesting that relatively specific olfactory sensitivity in the F1 generation was somehow inherited owing to F0 conditioning to that odor prior to conception. 
To study the structural representation of this process, we used M71-LacZ transgenic mice, wherein an acetophenoneresponsive olfactory sensory neuron population that expresses the M71 odorant receptor can be visualized. Using these mice, we demonstrated that the F1 male offspring of acetophenone-conditioned F0 males have more M71-expressing olfactory sensory neurons in the olfactory epithelium resulting in larger M71 glomeruli in the olfactory bulbs. Such enhanced M71 neuroanatomical representation was also observed when sperm (instead of normative mating) from acetophenone-conditioned F0 males was used to generate an IVF-derived F1 generation. These data suggest that the structure and behavioral olfactory sensitivity effects observed were inherited through the gametes (Figure 3).

When F1 odor-naïve males that had an F0 generation conditioned with acetophenone or propanol were mated with naïve females, the F2 generation also had an enhanced sensitivity to the F0 conditioned odor. The F2 generation of acetophenone-conditioned F0 males also had enhanced M71 representation. The behavioral and neuroanatomical effects persist even after cross-fostering studies in exposed dams.

These convergent lines of evidence from IVF, crossfostering, and F2 studies led us to conclude that the effects we observed were indeed biologically inherited and not merely socially transmitted. We next began to examine possible mechanisms underlying these effects. By virtue of our experimental design with specific odors, we adopted a candidate gene approach to investigate gene regulation of the M71 receptor locus. Bisulfite sequencing of the M71 locus from sperm DNA of both the F0 and F1 generations indicated hypo-methylation at this gene, potentially setting it up for enhanced transcription as is evidenced by the enhanced M71 representation.

Our working hypothesis, combining our data with that of the Bale lab, is that repeated odor fear conditioning in the ancestral generation, perhaps via small non-coding RNA transmitted via the vasculature, leads to decreased methylation of odor-specific receptor genes in the gametes. These epigenetic marks then, via an unknown mechanism, escape reprogramming during F1 development, allowing M71 odorant receptors to be expressed, or chosen, more readily in developing olfactory sensory neurons within the nose. An increased number of M71 neurons during F1 development would then support larger M71 glomeruli and increased M71-odorant-specific sensitivity in the next generation.

All these studies taken together make a compelling case for being able to model the effect of paternal environmental experiences on behavior, physiology, and neuroanatomy in future generations. By way of mechanisms, non-coding RNA species like miRNA and other epigenetic modifications like DNA methylation may emerge as candidates for intergenerational and transgenerational inheritance of characteristics. However, it is important to note that stressors likely act on different epigenetic mechanisms as a function of the developmental stage of the germ cells. Changes in DNA methylation, for example, require a complex machinery of specific proteins to read, write, and erase DNA methylation marks during the early phases of spermatogenesis that are not present in later developmental stages. Mechanisms such as loading small RNAs into adult sperm cells may be relevant in later developmental stages and may lead to distinct phenotypic outcomes.

\section{RELEVANCE AND IMPLICATIONS OF INTERGENERATIONAL AND TRANSGENERATIONAL TRANSMISSION OF DISEASE RISK}

The immediate transfer of information related to environmental conditions from ancestors to subsequent generations would be a highly beneficial mechanism to adapt to changing environments without the need for costly and timeconsuming DNA sequence adaptations. Changing environments with regard to reproduction, nutritional resources, and survival in threatening situations would therefore benefit from both behavioral and non-genetic transfer of information leading to an advantage in natural selection beyond genetic means.

The suggested mechanisms underlying this transfer are as divergent as the developmental stages at which the ancestral environment can influence the offspring generation. Here, we highlighted three broadly defined periods, (i) postnatal development, (ii) in utero development, and (iii) the preconception period, by reviewing selected examples from each period. This clearly does not exclude other, more specific periods in development that may allow the transfer of defined information between generations potentially resulting in different molecular and behavioral outcomes. This also applies to the molecular mechanisms that we are currently starting to understand. In utero programming of the F1 generation, the developing F2 primordial germ cells in F1 animals, and pre-conception exposure of the gametes all provide a direct exposure that may be explained through mechanisms of epigenetic programming; however, it remains largely unclear how specificity of such marks is achieved and how they can escape the broad erasure, or reprogramming, of epigenetic modifications during development.

The transmission of information by behavioral and social means may be the easiest to explain. As outlined earlier, maternal behavior in rodents triggers long-lasting epigenetic, hormonal, and likely learned behavioral mechanisms in the offspring generation. The plasticity of the epigenome, especially in early development, facilitates this programming through environmental exposure, and may serve as one form of genomic long-term memory that directly influences survival and reproductive behavior in the next generation. A biological transmission via epigenetic marks between both generations is therefore not necessary for this method of information transfer. Such a mode of transmission has been shown for non-human primates as well, where maltreatment of offspring by aggressive mothers runs in families and can be interrupted by cross-fostering to non-abusive foster mothers (Maestripieri, 2005). Similar observations can be 
made in humans, with individuals who experienced maltreatment in childhood being at higher risk to adopt such behaviors toward their own children (Ertem et al, 2000). These examples highlight the importance of epigenetic programming of behaviors in early life through the behavior of the parental generation and highlight the long-term impact of severe stress on the present and future generation.

Translated to human psychiatric disorders, this discussion highlights two important things. The early life environment provided by society for the next generations with regard to stress and abuse exposure, but also socioeconomic status, parental care, nutritional, emotional, and educational supply, all may strongly influence disease development and prevalence. Instead of treating complex and chronic psychiatric disorders, often unsuccessfully, in adulthood with increasing economic and medical requirements, early intervention would offer an eminent opportunity to prevent psychiatric disorders by providing supportive, adequate environments. This highlights another important factor. The plasticity of the epigenome especially, but not exclusively, in the early phases of development may offer therapeutic windows for disorders that have genetic and environmental components, as is seen in stress-related and anxiety disorders. Here, trajectories toward health and disease may be more plastic and not yet determined, in comparison with later stages of life that may require much more effort to reopen and remodel epigenetic programs.

More evidence is accumulating for a biological inheritance of environmentally acquired epigenetic marks in mammals. This is now not particularly controversial in other fields such as plant development, where there has been more progress in understanding the epigenome. But in mammals, with regard to trait inheritance, the possibility that an environmental exposure in humans can affect subsequent generations even without a direct exposure has triggered many controversial discussions (Grossniklaus et al, 2013). Although some studies have provided a glimpse into the underlying mechanisms, this is a largely unexplored field. Below we further explore the question, "how can an epigenetic trait be inherited'? Nonetheless, both maternal and paternal inheritance is documented, the majority of animal studies focus on the paternal line most likely owing to technical reasons and the availability of male sperm cells.

\section{INHERITANCE OF INFORMATION THROUGH EPIGENETIC MECHANISMS}

The accessibility of the DNA through chromatin remodeling is central to the regulation of gene transcription, and epigenetic mechanisms control the transcriptional regulation without changing the underlying DNA sequence. These mechanisms include the posttranslational modification of histone proteins and modifications of single nucleotides, most commonly in the form of DNA methylation or hydroxymethylation at cytosine residues, thereby altering the chromatin structure and the accessibility of the DNA to transcriptional regulators. In addition, the regulation of transcription and translation by non-coding RNAs can be regarded as an additional part of the epigenetic machinery. Text Box 1 summarizes the main features of epigenetic mechanisms relevant for this review (Jenuwein and Allis, 2001; Jaenisch and Bird, 2003; Holliday, 2006; Egger et al, 2004; Bird, 2007; Bonasio et al, 2010; Peschansky and Wahlestedt, 2014).

Important to the discussion of epigenetic inheritance is the term 'epiallele' that is defined as distinguishable variation of the same genetic locus with regard to its epigenetic characteristics and thus activity (Dolinoy et al, 2007). The agouti mouse model is one well-understood example of epigenetic transmission. The murine yellow agouti allele $\left(\mathrm{A}^{\mathrm{vy}}\right)$ can be regarded as the prototype example for an epiallele that results in a yellow to brown coat color as function of variable degrees of DNA methylation at a genetically stable IAP retrotransposon element (Waterland and Jirtle, 2003). The DNA methylation at the $\mathrm{A}^{\mathrm{vy}}$ locus is sensitive to environmental conditions during pregnancy in form of dietary supplementation with methyl donors leading to variations in DNA methylation at the $\mathrm{A}^{\mathrm{vy}}$ locus and subsequent coat color in the offspring generation. In human studies, the definition of epiallele is often not followed in a strict sense but is applied to loci that differ in the underlying genomic sequence, most often by single nucleotide polymorphisms influencing gene activity through allele-dependent epigenetic mechanisms (Klengel et al, 2013b). Current evidence likely underestimates the influence of small genetic variations on DNA methylation, potentially accounting for a large portion of epigenetic differences observed in current studies (Bell et al, 2011).

\section{REGULATION OF DNA METHYLATION AND ESCAPE FROM REPROGRAMMING}

In contrast to early concepts, DNA methylation predominantly at palindromic cytosine-guanine dinucleotides is a dynamic epigenetic mechanism that regulates chromatin structure though influencing DNA-binding proteins. Importantly, this modification is heritable with DNA methyltransferase 1 (DNMT1) maintaining the double-stranded methylation pattern through binding at hemi-methylated DNA found after DNA replication. However, former evidence suggested that DNA methylation is completely erased during development of the primordial germ cells and during fertilization with variable timelines between the paternal and maternal genomes. However, this erasure, or reprogramming, is not complete. Evidence for certain loci that escape reprogramming is growing with examples including imprinted genes and repetitive elements (Kobayashi et al, 2012; Radford et al, 2014; Tang et al, 2015). It is conceivable that other loci may escape the complete erasure as well; furthermore, the idea that the numerous repetitive elements present in the genome can transfer epigenetic information through differential 
methylation is an interesting option especially because these elements have the potential to influence the expression of genes. Studies that we used as examples above also included the investigation of DNA methylation in male germ cells, which have found promising differences in response to the environmental factors used. However, subsequent studies are needed to determine the stability of these marks across the reprogramming process and to address the heterogeneity in methylation between germ cells as well as the influence on the individual methylation level in the progeny. In addition, it remains unclear how DNA methylation in germ cells and the developing organism is directed to the relevant cell type, eg, neurons within a multicellular organism derived from a totipotent cell, or whether the DNA methylation marks would remain present in most somatic tissues.

\section{REGULATION OF HISTONE MODIFICATIONS AND ESCAPE FROM REPROGRAMMING}

Less evidence exists for the transmission of epigenetic information through histone modifications. The majority of histone proteins in sperm are replaced by protamines to facilitate DNA condensation and stabilization. However, a substantial portion of $10-15 \%$ (human) and about $1 \%$ (mouse) of sperm DNA is packed by histones, facilitating the transcriptional activation of genes necessary in early development (Balhorn, 2007; Brykczynska et al, 2010). Histone modifications, although similarly erased as DNA methylation, can escape reprogramming and thus provide an opportunity for the inheritance of information. Although not explicitly outlined in the given examples, there is evidence for transmission of epigenetic information relevant to the field of psychiatry through the sperm histone code. Vassoler et al (2013) investigated the effect of ancestral cocaine selfexposure on subsequent generations and found that male offspring from cocaine sires developed a delayed and reduced cocaine self-administration phenotype. This behavior was linked to increased BDNF transcription and translation in the medial prefrontal cortex with an increase in permissive acetylated histone $\mathrm{H} 3$ at the BDNF promoter regions. A stronger association of acetylated histone $\mathrm{H} 3$ with the BDNF promoters was also found in sperm of cocaine self-administrating F0 animals, suggesting that the histone/ protamine code in fact is not completely erased and may carry environmental information from the ancestral generation. However, it remains an open question if the altered histone modification pattern in F0 sperm is directly linked to the molecular and behavioral outcomes in F1 or if other, interconnected epigenetic mechanisms are responsible for the inheritance of this phenotype.

\section{NON-CODING RNA MECHANISMS}

More recently, the focus of potential mechanisms shifted toward non-coding RNAs. However, our understanding of the biology of non-coding transcripts that makes up the largest portion of the genome-wide transcriptional output is limited. Examples also included in this review highlight the potential of miRNAs that can influence posttranscriptional transcript stability and translation in the cell. First and foremost, the study by Gapp et al (2014), makes a compelling case for the function of RNAs in sperm to influence the phenotype of the subsequent generation. Identified by an unbiased sequencing approach, the authors found miRNAs that are enriched in the sperm of MSUS mice. Injecting purified RNA from the sperm of MSUS mice into naïve fertilized eggs lead to offspring that resembled the previously defined phenotype. It is conceivable that these miRNAs survive reprogramming and influence the genome of the developing offspring by inducing more long-lasting epigenetic imprints. However, the authors also noted differentially expressed piRNA (PIWI-interacting RNA) clusters. PiRNAs are considered to have an important role in silencing retrotransposons and thus may influence gene expression in subsequent generations through epigenetic modifications of repetitive elements.

The excitement about the biology of small RNAs is not only founded on the intriguing mechanisms of transcriptional regulation, but also on more recent evidence for small RNAs as a carrier of information throughout the body. Stress in general will affect multiple tissues and may include the germ cells via direct effects. However, very specific signals that are presumably not system-wide need a signaling cascade from one tissue to the next, eg, the brain to the sperm. Small RNAs potentially packed into carrier units such as exosomes (small lipid vesicles released, for example, from lipid rafts from cells into plasma) might be able to function as long-range signals to the gametes, provided that germ cells can actively screen their environment for these signals. Although fascinating, these concepts clearly are in need of future research and experimental evidence for validation, verification, and identifying the next direction of inquiry.

\section{FUTURE DIRECTIONS AND CLINICAL IMPLICATIONS}

Risk for psychopathology is a multigenerational phenomenon, with a genetic component inherited from the parents and an environmental component that may be epigenetically inherited or behaviorally transmitted from the ancestral generation to the offspring. Beyond controversy, the behavior of the parental generation can influence the risk for the development of psychiatric disorders in the progeny from fetal development onwards. Such parental transmission of risk surely includes parenting behavior, shared genetics, and shared risk environments. Additionally, human studies investigating such effects point toward the detrimental effects of in utero exposure with multiple risks in the subsequent generation, eg, depression during pregnancy, socioeconomic deprivation, stress exposure, toxicants, and obesity. This also applies for studies on postnatal and early life stress that is characterized by the long-lasting influence of detrimental 
stressors such as childhood abuse on mental health trajectories.

Rodent studies enable us to model the effect of environmental influence on behavioral and molecular marks at different developmental stages while controlling for confounders that are inevitable in human studies. Both fascinating and controversial studies now suggest that biological mechanisms may shape the offspring's risk to disease through the inheritance of epigenetic modifications even across several generations. However, a simple extrapolation of the results found in rodents to humans is not possible, and it remains speculative at the moment whether the biological inheritance of environmentally acquired marks is truly relevant for the development of psychiatric diseases in humans. Nevertheless, the rodent models illustrated here offer invaluable insight into the molecular biology of the perception of environmental conditions, the long-term programming of relevant signaling cascades, and the inheritance and transmission of such information to subsequent generations.

Future studies need to unequivocally establish the molecular mechanisms of epigenetic inheritance by investigating the effect of environmental factors on the germ cells and the potential mode of transmission of information from the brain to the gametes. In addition, environmental effects directly influencing in utero and postnatal development through epigenetics will highlight trajectories to disease and resilience, leading to new preventive and therapeutic approaches that may help to interrupt the cycle of environmentally induced stress and anxiety disorders across generations.

\section{FUNDING AND DISCLOSURE}

The authors declare no conflict of interest.

\section{ACKNOWLEDGMENTS}

We thank Tracy Bale and Ali Rodgers for sharing unpublished results. Research in the Ressler lab is supported by the Howard Hughes Medical Institute, NIH (R01MH096764) and by an NIH/NCRR base grant (P51RR000165) to the Yerkes National Primate Research Center. TK is supported by an EMBO long-term fellowship (1153-2013) and a NARSAD Young Investigator Grant (YI 20895).

\section{REFERENCES}

Balhorn R (2007). The protamine family of sperm nuclear proteins. Genome Bio/ 8: 227.

Bell JT, Pai AA, Pickrell JK, Gaffney DJ, Pique-Regi R, Degner JF et al (2011). DNA methylation patterns associate with genetic and gene expression variation in HapMap cell lines. Genome Biol 12: 13.

Bierer LM, Bader HN, Daskalakis NP, Lehrner AL, Makotkine I, Seckl JR et al (2014). Elevation of $11 \beta$-hydroxysteroid dehydrogenase type 2 activity in Holocaust survivor offspring: evidence for an intergenerational effect of maternal trauma exposure. Psychoneuroendocrinology 48: 1-10.

Bird A (2007). Perceptions of epigenetics. Nature 447: 396-398.
Bonasio R, Tu S, Reinberg D (2010). Molecular signals of epigenetic states. Science 330: 612-616.

Brykczynska U, Hisano M, Erkek S, Ramos L, Oakeley EJ, Roloff TC et al (2010). Repressive and active histone methylation mark distinct promoters in human and mouse spermatozoa. Nat Struct Mol Biol 17: 679-687.

Carone BR, Fauquier L, Habib N, Shea JM, Hart CE, Li R et al (2010). Paternally induced transgenerational environmental reprogramming of metabolic gene expression in mammals. Cell 143: 1084-1096.

Champagne FA (2008). Epigenetic mechanisms and the transgenerational effects of maternal care. Front Neuroendocrinol 29: 386-397.

Champagne FA, Meaney MJ (2006). Stress during gestation alters postpartum maternal care and the development of the offspring in a rodent model. Biol Psychiatry 59: 1227-1235.

Compton WM, Conway KP, Stinson FS, Grant BF (2006). Changes in the prevalence of major depression and comorbid substance use disorders in the United States between 1991-1992 and 2001-2002. Am J Psychiatry 163: 2141-2147.

Crews D, Gore AC, Hsu TS, Dangleben NL, Spinetta M, Schallert T et al (2007). Transgenerational epigenetic imprints on mate preference. Proc Natl Acad Sci USA 104: 5942-5946.

Daxinger L, Whitelaw E (2012). Understanding transgenerational epigenetic inheritance via the gametes in mammals. Nat Rev Genet 13: 153-162.

Dias BG, Ressler KJ (2014a). Experimental evidence needed to demonstrate interand trans-generational effects of ancestral experiences in mammals. BioEssays 36: 919-923.

Dias BG, Ressler KJ (2014b). Parental olfactory experience influences behavior and neural structure in subsequent generations. Nat Neurosci 17: 89-96.

Dietz DM, LaPlant Q, Watts EL, Hodes GE, Russo SJ, Feng J et al (2011). Paternal Transmission of Stress-Induced Pathologies. Biol Psychiatry 70: 408-414.

Dolinoy DC, Das R, Weidman JR, Jirtle RL (2007). Metastable epialleles, imprinting, and the fetal origins of adult diseases. Pediatr Res 61(Pt 2): 30r-37r.

Egger G, Liang G, Aparicio A, Jones PA (2004). Epigenetics in human disease and prospects for epigenetic therapy. Nature 429: 457-463.

Ertem IO, Leventhal JM, Dobbs S (2000). Intergenerational continuity of child physical abuse: how good is the evidence? Lancet 356: 814-819.

Flory JD, Bierer LM, Yehuda R (2011). Maternal exposure to the holocaust and health complaints in offspring. Dis Markers 30: 133-139.

Francis D, Diorio J, Liu D, Meaney MJ (1999). Nongenomic transmission across generations of maternal behavior and stress responses in the rat. Science 286: 1155-1158.

Franklin TB, Russig H, Weiss IC, Graff J, Linder N, Michalon A et al (2010). Epigenetic transmission of the impact of early stress across generations. Biol Psychiatry 68: 408-415.

Gapp K, Jawaid A, Sarkies P, Bohacek J, Pelczar P, Prados J et al (2014). Implication of sperm RNAs in transgenerational inheritance of the effects of early trauma in mice. Nat Neurosci 17: 667-669.

Gillespie CF, Bradley B, Mercer K, Smith AK, Conneely K, Gapen M et al (2009). Trauma exposure and stress-related disorders in inner city primary care patients. Gen Hosp Psychiatry 31: 505-514.

Gillette R, Miller-Crews I, Nilsson EE, Skinner MK, Gore AC, Crews D (2014). Sexually dimorphic effects of ancestral exposure to vinclozolin on stress reactivity in rats. Endocrinology 155: 3853-3866.

Goodman SH, Gotlib IH (1999). Risk for psychopathology in the children of depressed mothers: a developmental model for understanding mechanisms of transmission. Psychol Rev 106: 458-490.

Grossniklaus U, Kelly WG, Ferguson-Smith AC, Pembrey M, Lindquist S (2013). Transgenerational epigenetic inheritance: how important is it? Nat Rev Genet 14: 228-235.

Guffanti G, Galea S, Yan L, Roberts AL, Solovieff N, Aiello AE et al (2013). Genomewide association study implicates a novel RNA gene, the lincRNA AC068718.1, as a risk factor for post-traumatic stress disorder in women. Psychoneuroendocrinology 38: 3029-3038

Hauser J, Feldon J, Pryce CR (2009). Direct and dam-mediated effects of prenatal dexamethasone on emotionality, cognition and HPA axis in adult Wistar rats. Horm Behav 56: 364-375.

Heard E, Martienssen RA (2014). Transgenerational epigenetic inheritance: myths and mechanisms. Cell 157: 95-109.

Heijmans BT, Tobi EW, Stein AD, Putter H, Blauw GJ, Susser ES et al (2008). Persistent epigenetic differences associated with prenatal exposure to famine in humans. Proc Natl Acad Sci USA 105: 17046-17049.

Heimberg RG, Stein MB, Hiripi E, Kessler RC (2000). Trends in the prevalence of social phobia in the United States: a synthetic cohort analysis of changes over four decades. Eur Psychiatry 15: 29-37.

Hidaka BH (2012). Depression as a disease of modernity: explanations for increasing prevalence. J Affect Disord 140: 205-214. 
Hoge CW, Castro CA, Messer SC, McGurk D, Cotting DI, Koffman RL (2004). Combat duty in Iraq and Afghanistan, mental health problems, and barriers to care. N Engl J Med 351: 13-22.

Holliday R (2006). Epigenetics: a historical overview. Epigenetics 1: 76-80.

Jaenisch R, Bird A (2003). Epigenetic regulation of gene expression: how the genome integrates intrinsic and environmental signals. Nat Genet 33: 245-254.

Jenuwein T, Allis CD (2001). Translating the histone code. Science 293: 1074-1080. Kaati G, Bygren LO, Pembrey M, Sjostrom M (2007). Transgenerational response to nutrition, early life circumstances and longevity. Eur J Hum Genet 15: 784-790.

Kendler KS, Bulik CM, Silberg J, Hettema JM, Myers J, Prescott CA (2000). Childhood sexual abuse and adult psychiatric and substance use disorders in women: an epidemiological and cotwin control analysis 23 173. Arch Gen Psychiatry 57: 953-959.

Kessler RC, Chiu WT, Demler O, Merikangas KR, Walters EE (2005). Prevalence, severity, and comorbidity of 12-month DSM-IV disorders in the National Comorbidity Survey Replication. Arch Gen Psychiatry 62: 617-627.

Kessler RC, Davis CG, Kendler KS (1997). Childhood adversity and adult psychiatric disorder in the US National Comorbidity Survey. Psychol Med 27: 1101-1119.

Khashan AS, Abel KM, McNamee R, Pedersen MG, Webb RT, Baker PN et al (2008). Higher risk of offspring schizophrenia following antenatal maternal exposure to severe adverse life events. Arch Gen Psychiatry 65: 146-152.

Kinney DK, Miller AM, Crowley DJ, Huang E, Gerber E (2008). Autism prevalence following prenatal exposure to hurricanes and tropical storms in Louisiana. J Autism Dev Disord 38: 481-488.

Klengel T, Binder EB (2013a). Gene-environment interactions in major depressive disorder. Canad J Psychiatry 58: 76-83.

Klengel T, Mehta D, Anacker C, Rex-Haffner M, Pruessner JC, Pariante CM et al (2013b). Allele-specific FKBP5 DNA demethylation mediates gene-childhood trauma interactions. Nat Neurosci 16: 33-U59.

Kobayashi H, Sakurai T, Imai M, Takahashi N, Fukuda A, Yayoi O et al Contribution of intragenic DNA methylation in mouse gametic DNA methylomes to establish oocyte-specific heritable marks. PLoS Genet 2012; 8: e1002440.

Kriaucionis S, Heintz N (2009). The nuclear DNA base 5-hydroxymethylcytosine is present in Purkinje neurons and the brain. Science 324: 929-930.

Lee SH, Ripke S, Neale BM, Faraone SV, Purcell SM, Perlis RH et al (2013). Genetic relationship between five psychiatric disorders estimated from genomewide SNPs. Nat Genet 45: 984-994.

Lehrner A, Bierer LM, Passarelli V, Pratchett LC, Flory JD, Bader HN et al (2014). Maternal PTSD associates with greater glucocorticoid sensitivity in offspring of Holocaust survivors. Psychoneuroendocrinology 40: 213-220.

Levinson DF, Mostafavi S, Milaneschi Y, Rivera M, Ripke S, Wray NR et al (2014). Genetic studies of major depressive disorder: why are there no genome-wide association study findings and what can we do about it? Biol Psychiatry 76: 510-512.

Logue MW, Baldwin C, Guffanti G, Melista E, Wolf EJ, Reardon AF et al (2013). A genome-wide association study of post-traumatic stress disorder identifies the retinoid-related orphan receptor alpha (RORA) gene as a significant risk locus. Mol Psychiatry 18: 937-942.

Ma DK, Jang MH, Guo JU, Kitabatake Y, Chang ML, Pow-Anpongkul N et al (2009). Neuronal activity-induced Gadd45b promotes epigenetic DNA demethylation and adult neurogenesis. Science 323: 1074-1077.

Maestripieri D (2005). Early experience affects the intergenerational transmission of infant abuse in rhesus monkeys. Proc Natl Acad Sci USA 102: 9726-9729.

Major Depressive Disorder Working Group of the Psychiatric GWAS Consortium (2012). A mega-analysis of genome-wide association studies for major depressive disorder. Mol Psychiatry 18: 497-511.

Malanga CJ, Pejchal M, Kosofsky BE (2007). Prenatal exposure to cocaine alters the development of conditioned place-preference to cocaine in adult mice. Pharmacol Biochem Behav 87: 462-471.

Manuck SB, McCaffery JM. Gene-environment interaction. Annu Rev Psychol 2014; 65: 41-70.

McGowan PO, Suderman M, Sasaki A, Huang TCT, Hallett M, Meaney MJ et al (2011). Broad epigenetic signature of maternal care in the brain of adult rats. Plos One 6: e14739.

Mercer TR, Mattick JS (2013). Structure and function of long noncoding RNAs in epigenetic regulation. Nat Struct Mol Biol 20: 300-307.

Moisiadis VG, Matthews SG (2014a). Glucocorticoids and fetal programming part 1: outcomes. Nat Rev Endocrinol 10: 391-402.

Moisiadis VG, Matthews SG (2014b). Glucocorticoids and fetal programming part 2: mechanisms. Nat Rev Endocrinol 10: 403-411.

Morgan CP, Bale TL (2011). Early prenatal stress epigenetically programs dysmasculinization in second-generation offspring via the paternal lineage. J Neurosci 31: 11748-11755.
Morris KV, Mattick JS (2014). The rise of regulatory RNA. Nat Rev Genet 15 423-437.

Mueller BR, Bale TL (2007). Early prenatal stress impact on coping strategies and learning performance is sex dependent. Physiol Behav 91: 55-65.

Mueller BR, Bale TL (2008). Sex-specific programming of offspring emotionality after stress early in pregnancy. J Neurosci 28: 9055-9065.

Muhleisen TW, Leber M, Schulze TG, Strohmaier J, Degenhardt F, Treutlein J et al (2014). Genome-wide association study reveals two new risk loci for bipolar disorder. Nat Commun 5: 3339

Murr R. Interplay between different epigenetic modifications and mechanisms. Adv Genet 2010; 70: 101-141.

Nagano M, Ozawa H, Suzuki H (2008). Prenatal dexamethasone exposure affects anxiety-like behaviour and neuroendocrine systems in an age-dependent manner. Neurosci Res 60: 364-371.

Ng SF, Lin RC, Laybutt DR, Barres R, Owens JA, Morris MJ (2010). Chronic high-fat diet in fathers programs beta-cell dysfunction in female rat offspring. Nature 467: 963-966.

Nievergelt CM, Maihofer AX, Mustapic M, Yurgil KA, Schork NJ, Miller MW et al (2015). Genomic predictors of combat stress vulnerability and resilience in U.S. Marines: a genome-wide association study across multiple ancestries implicates PRTFDC1 as a potential PTSD gene. Psychoneuroendocrinology 51: 459-471.

Noorlander CW, Visser GH, Ramakers GM, Nikkels PG, de Graan PN (2008). Prenatal corticosteroid exposure affects hippocampal plasticity and reduces lifespan. Dev Neurobiol 68: 237-246.

Oliveira M, Rodrigues AJ, Leao P, Cardona D, Pego JM, Sousa N (2012). The bed nucleus of stria terminalis and the amygdala as targets of antenatal glucocorticoids: implications for fear and anxiety responses. Psychopharmacology (Berl) 220: 443-453.

Peschansky VJ, Wahlestedt C (2014). Non-coding RNAs as direct and indirect modulators of epigenetic regulation. Epigenetics 9: 3-12.

Radford EJ, Ito M, Shi H, Corish JA, Yamazawa K, Isganaitis E et al (2014). In utero effects. In utero undernourishment perturbs the adult sperm methylome and intergenerational metabolism. Science 345: 1255903.

Rodgers AB, Morgan CP, Bronson SL, Revello S, Bale TL (2013). Paternal stress exposure alters sperm microRNA content and reprograms offspring HPA stress axis regulation. J Neurosci 33: 9003-9012.

Rodgers AB, Morgan CP, Chan JC, Bale TL. MicroRNA in sperm: a causal linkbetween paternal experience and offspring stress regulation. 35012/NN18 Neuroscience 2014 Abstracts, Society for Neuroscience: Washington, DC, 2014.

Schizophrenia Working Group of the Psychiatric Genomics Consortium (2014). Biological insights from 108 schizophrenia-associated genetic loci. Nature 511 : 421-427.

Seckl JR, Meaney MJ (2006). Glucocorticoid "programming" and PTSD risk. Ann NY Acad Sci 1071: 351-378.

Skinner MK (2008). What is an epigenetic transgenerational phenotype? F3 or F2. Reprod Toxicol 25: 2-6.

Skinner MK, Anway MD, Savenkova MI, Gore AC, Crews D (2008). Transgenerational epigenetic programming of the brain transcriptome and anxiety behavior. PLoS One 3: e3745.

Suderman M, McGowan PO, Sasaki A, Huang TCT, Hallett MT, Meaney MJ et al (2012). Conserved epigenetic sensitivity to early life experience in the rat and human hippocampus. Proc Natl Acad Sci USA 109: 17266-17272.

Sullivan PF, Daly MJ, O'Donovan M (2012). Genetic architectures of psychiatric disorders: the emerging picture and its implications. Nat Rev Genet 13: 537-551.

Sweatt JD (2013). The emerging field of neuroepigenetics. Neuron 80: 624-632.

Szulwach KE, Li X, Li Y, Song CX, Wu H, Dai Q et al (2011). 5-hmC-mediated epigenetic dynamics during postnatal neurodevelopment and aging. Nat Neurosci 14: 1607-1616.

Tang WW, Dietmann S, Irie N, Leitch HG, Floros VI, Bradshaw CR et al A unique gene regulatory network resets the human germline epigenome for development. Cell 2015; 161: 1453-1467.

Tobi EW, Goeman JJ, Monajemi R, Gu H, Putter H, Zhang Y et al (2014). DNA methylation signatures link prenatal famine exposure to growth and metabolism. Nat Commun 5: 5592.

Todrank J, Heth G, Restrepo D (2011). Effects of in utero odorant exposure on neuroanatomical development of the olfactory bulb and odour preferences. Proc Biol Sci 278: 1949-1955.

van Os J, Selten JP (1998). Prenatal exposure to maternal stress and subsequent schizophrenia. The May 1940 invasion of The Netherlands. Br J Psychiatry 172: 324-326.

Vassoler FM, White SL, Schmidt HD, Sadri-Vakili G, Pierce RC (2013). Epigenetic inheritance of a cocaine-resistance phenotype. Nat Neurosci 16: 42-47. 
Wang W, Nitulescu I, Lewis JS, Lemos JC, Bamford IJ, Posielski NM et al (2013). Overinhibition of corticostriatal activity following prenatal cocaine exposure. Ann Neurol 73: 355-369.

Waterland RA, Jirtle RL (2003). Transposable elements: targets for early nutritional effects on epigenetic gene regulation. Mol Cell Biol 23: 5293-5300.

Weaver IC, Cervoni N, Champagne FA, D'Alessio AC, Sharma S, Seckl JR et al (2004). Epigenetic programming by maternal behavior. Nat Neurosci 7: 847-854.

Whiteford HA, Degenhardt L, Rehm J, Baxter AJ, Ferrari AJ, Erskine HE et al (2013). Global burden of disease attributable to mental and substance use disorders: findings from the Global Burden of Disease Study 2010. Lancet 382: 1575-1586.

Wittchen HU, Jacobi F, Rehm J, Gustavsson A, Svensson M, Jonsson B et al (2011). The size and burden of mental disorders and other disorders of the brain in Europe 2010. Eur Neuropsychopharmacol 21: 655-679.

Wray NR, Pergadia ML, Blackwood DH, Penninx BW, Gordon SD, Nyholt DR et al (2012). Genome-wide association study of major depressive disorder: new results, meta-analysis, and lessons learned. Mol Psychiatry 17: 36-48.
Yehuda R, Bell A, Bierer LM, Schmeidler J (2008). Maternal, not paternal, PTSD is related to increased risk for PTSD in offspring of Holocaust survivors. J Psychiatr Res 42: 1104-1111.

Yehuda R, Daskalakis NP, Lehrner A, Desarnaud F, Bader HN, Makotkine I et al Influences of maternal and paternal PTSD on epigenetic regulation of the glucocorticoid receptor gene in Holocaust survivor offspring. Am J Psychiatry 2014; 171: 872-880.

Yehuda R, Engel SM, Brand SR, Seckl J, Marcus SM, Berkowitz GS (2005). Transgenerational effects of posttraumatic stress disorder in babies of mothers exposed to the World Trade Center attacks during pregnancy. J Clin Endocrinol Metab 90: 4115-4118.

Zhang TY, Labonte B, Wen XL, Turecki G, Meaney MJ (2013). Epigenetic mechanisms for the early environmental regulation of hippocampal glucocorticoid receptor gene expression in rodents and humans. Neuropsychopharmacology 38: 111-123.

Zuloaga DG, Carbone DL, Quihuis A, Hiroi R, Chong DL, Handa RJ (2012). Perinatal dexamethasone-induced alterations in apoptosis within the hippocampus and paraventricular nucleus of the hypothalamus are influenced by age and sex. $J$ Neurosci Res 90: 1403-1412. 